\title{
Efeitos de terapêuticas respiratórias e actividade física nas pressões respiratórias máximas de mulheres obesas
}

\author{
Effects of respiratory therapeutics and physical activity in maximal \\ respiratory pressures of obese women
}

\author{
M.S.L. Cruz, P. Roquetti Fernandes, E. Sonehara, V.M. Reis, F.B. Policarpo, J. Fernandes \\ Filho
}

RESUMO

O estudo teve por objectivo verificar os efeitos de diferentes terapêuticas nas pressões respiratórias estáticas máximas em mulheres obesas. Para o efeito, 51 mulheres obesas foram divididas em três grupos: i) sedentárias $(n=19)$ com $57.37 \pm 8.10$ anos e IMC $=38.3 \pm 5.9$; ii $)$ fisicamente ativas $(n=$ 13) com idade de $60.31 \pm 8.94$ anos e IMC $=38.0 \pm 7.7$, as quais foram submetidas a sete dias de terapêutica com incentivador respiratório; e, iii) pacientes de reabilitação pulmonar $(n=20)$ com idade de $48.75 \pm 13.71$ anos e IMC $=34.7 \pm 3.8$, que participaram de um programa de 12 semanas de actividade física aeróbia a $70 \%$ da frequência cardíaca de reserva, sessões de exercícios respiratórios e alongamentos. A análise comparativa foi por meio da one way ANOVA com Post-Hoc de Turkey. Para a comparação intra-grupos aplicou-se o teste $\mathrm{t}$ emparelhado. A determinação do coeficiente de correlação foi por meio do teste Pearson. Os resultados obtidos descrevem alterações significativas pósterapêutica $(p<.05)$ para as pressões respiratórias estáticas máximas entre e intra-grupo. Concluiu-se que as terapêuticas aplicadas promoveram modificações significativas nas pressões respiratórias estáticas máximas em mulheres obesas.

Palavras-chave: reabilitação pulmonar, actividade física, pressão respiratória, obesas

ABSTRACT

The aim of this study was to evaluate the effects of differing therapies on maximal static respiratory pressure in 51 obese women divided into three groups: i) sedentary women $(n=19$, aged $57.37 \pm$ 8.10 years, BMI $=38.3 \pm 5.9)$; ii $)$ physically active women $(n=13$, aged $60.31 \pm 8.94$ years, BMI $=$ $38.0 \pm 7.7)$ submitted to seven days of therapy through incentive spirometry, and iii) women in pulmonary rehabilitation $(n=20$, aged $48.75 \pm 13.71$ years, BMI $=34.7 \pm 3.8)$ submitted to a 12 -week program of aerobic physical activity at $70 \%$ heart rate reserve, and breathing and stretching exercises. Data were treated using a one way ANOVA with post-hoc Turkey, within groups with paired test and Pearson's correlation coefficient. Results showed a significant post-therapy $(p<.05)$ for the maximal static respiratory pressure between and within group with significant changes on obese women's maximal static respiratory pressure.

Keywords: pulmonary rehabilitation, physical activity, respiratory pressure, obese

Submetido: 24.07.2009 | Aceite: 13.02.2010

Maria do Socorro Luna Cruz e Elisa Sonehara. Universidade Potiguar, Natal - RN, Brasil; Universidade Castelo Branco, Rio de Janeiro - RJ, Brasil.

Paula Roquetti Fernandes. Centro de Excelência de Avaliação Física, Rio de Janeiro RJ, Brasil.

Victor Machado Reis e Fernando Policarpo Barbosa. Universidade de Trás-os-Montes e Alto Douro, Vila Real Portugal; Centro de Investigação em Desporto, Saúde e Desenvolvimento Humano, Vila Real, Portugal.

José Fernandes Filho. Centro de Excelência de Avaliação Física, Rio de Janeiro RJ, Brasil; Universidade Federal do Rio de Janeiro, Rio de Janeiro - RJ, Brasil.

Endereço para correspondência: Maria do Socorro Luna Cruz, Universidade Potiguar - RN, Rua Marise Bastier, 275B - Ap. 803. Ed. Rui Feliciano. CEP. 59075-070 Natal - RN, Brasil.

E-mail: socorrolcruz@gmail.com 
A obesidade é uma comorbidade que está relacionada com enfermidades degenerativas do sistema circulatório e distúrbios metabólicos. De igual forma, o excesso de tecido adiposo promove complicações sobre o sistema respiratório (Guimarães, Avezum, \& Piegas, 2006; Rocca, Tirapegui, Melo, \& Ribeiro, 2008; Romero \& Zanesco, 2006), causando alterações negativas na mecânica ventilatória de indivíduos obesos. Vários são os autores que apontaram que as pressões estáticas máximas, inspiratória (PImáx) e expiratória (PEmáx) de obesos quando comparadas às de indivíduos não obesos é significativamente menor (Paisani, Chaivegato, \& Faresin, 2005).

A questão a ser respondida sobre a redução das capacidades respiratórias, em decorrência da adiposidade, é qual a estratégia que deve ser aplicada na reabilitação e na manutenção da integridade do sistema respiratório. Para Maeda (2004), a redução das capacidades pulmonares e da complacência se relaciona com o aumento da resistência gerada pelo tecido adiposo em torno das costelas, abdómen e diafragma. A sobrecarga, ocasionada na musculatura respiratória, provoca a necessidade de um esforço superior para manter a ventilação adequada. $\mathrm{O}$ facto anteriormente referido, é apontado por Domingos (2003) como umas das causas da necessidade de se estudar a mecânica ventilatória em homens obesos.

Entre os métodos que podem ser aplicados, na prevenção primária e/ou secundária das complicações respiratórias, destaca-se o uso da inspirometria de incentivo. A terapêutica, anteriormente referida, tem por objectivo estimular a mecânica respiratória, ocasionando melhorias na distribuição do volume pulmonar, devido ao fortalecimento da musculatura respiratória, permitindo, assim, incrementos ventilatórios na sua totalidade (Romanini et al., 2007). Ressalta-se que o método em voga está entre os procedimentos pré-operatório em cirurgia bariátrica ou como método preventivo para complicações pós- operatório torácicas e abdominais (Romanini et al., 2007).

Outra possível linha de intervenção é a utilização de programas multidisciplinares de reabilitação cardiopulmonar com o objectivo de promover mudanças comportamentais não procurando obter somente os seus efeitos agudos, estabelecendo, assim, um novo estilo de vida (Godoy, Teixeira Paulo, Becker Júnior, Michelli, \& Godoy, 2009). Embora o método de inspirometria de incentivo e os programas multidisciplinares de reabilitação cardiopulmunar tenham objectivos similares, há uma recuperação das capacidades funcionais em períodos distintos. $\mathrm{O}$ primeiro tem como finalidade aprimorar as capacidades respiratórias para procedimento cirúrgico, enquanto o outro procura atender aspectos mais amplos que levam à melhoria global do indivíduo portador de obesidade. Assim, o presente estudo teve por objectivo verificar os efeitos de diferentes terapêuticas nas pressões respiratórias estáticas máximas em mulheres obesas.

\section{MÉTODO}

O desenho do estudo aplicado foi do tipo quase-experimental, tendo uma característica longitudinal.

\section{Amostra}

O estudo contou com uma amostra de 51 mulheres obesas com idade entre 18 e 75 anos, voluntárias, divididas em três grupos: grupo 1: sedentárias (G1, $n=19$ ); grupo 2: fisicamente activas $(G 2, n=13)$ e grupo 3: reabilitação pulmonar $(\mathrm{G} 3, n=20)$. As voluntárias receberam os esclarecimentos pertinentes sobre os riscos e benefícios do projecto e foram convidadas a assinar o termo de consentimento livre esclarecido. Este estudo foi aprovado pelo comité de ética da instituição (protocolo $\mathrm{n}^{\circ}$ 0052/2007).

\section{Procedimentos}

Após a assinatura, as voluntárias foram submetidas a uma entrevista e à mensuração 
dos parâmetros massa corporal e estatura, usando uma balança digital (Toledo ${ }^{\circledR}$ - Brasil), com limite de $150 \mathrm{~kg}$ e acuidade de $50 \mathrm{~g}$, e um estadiómetro com a escala em centímetros, respectivamente. Através dos parâmetros, anteriormente referidos, foi determinado o índice de massa corporal (IMC).

Após a determinação dos parâmetros antropométricos, foram realizadas as mensurações da pressão inspiratória máxima (PImáx) e pressão expiratória máxima (PEmáx), com uso de um manovacuômetro (Gerar Ind., Brasil) com limite operacional de -300 a $+300 \quad \mathrm{cmH}_{2} \mathrm{O}$. Os testes foram executados estando o indivíduo na posição sentado, com o tronco formando um ângulo de $90^{\circ}$ com a articulação coxo femoral, de acordo com as directrizes da Sociedade Brasileira de Pneumologia e Tisiologia (SBPT, 2002). Para realização dos testes de função pulmonar, as narinas encontravam-se ocluídas com clipe nasal. Ao manovacuômetro estava acoplado uma traqueia de plástico de $15 \mathrm{~cm}$, à qual se encontrava encaixada o bocal. Os indivíduos foram instruídos sobre os procedimentos para execução das medidas, em seguida realizaram um treinamento para adaptação ao equipamento. Depois do devido entendimento do exame, por parte do indivíduo, foi realizada a mensuração. A PImáx foi medida com o indivíduo realizando uma expiração forçada, até o volume residual (VR), seguida de uma inspiração até a capacidade pulmonar total (CPT), sustentada por três segundos. Para a obtenção da PEmáx realizou-se uma inspiração profunda, até à $\mathrm{CPT}$, seguida de uma expiração forçada máxima até ao VR contra a via aérea ocluída, mantendo a mesma por três segundos. O coeficiente de variação admitido para a PImáx e PEmáx foi de $10.2 \%$ e $12.8 \%$, respectivamente (Parreira et al., 2007). Exigiuse que as pressões fossem mantidas estáveis durante dois segundos (SBPT, 2002). O intervalo de tempo entre as medidas foi determinado de acordo com as necessidades de cada indivíduo, porém com o mínimo de trinta segundos entre uma manobra e outra. Para a análise dos dados foi considerado apenas o maior valor aferido para cada PImáx e PEmáx, descartando-se os outros valores.

\section{Protocolo de Intervenção}

Após os procedimentos de avaliação, todos os grupos foram submetidos aos protocolos de intervenção. Para o G1 e G2 foi aplicada a terapêutica do incentivador respiratório por um período de 7 dias, realizando três vezes ao dia, com três séries de dez repetições, totalizando 90 repetições diárias (Azeredo, 2000; Godoy et al., 2009). Para o efeito, os voluntários levaram o incentivador respiratório para casa e no final do protocolo proposto entregaram a ficha de controle. O G3 foi submetido a um programa de treino composto de exercícios aeróbios com intensidade de $70 \%$ da frequência cardíaca de reserva e exercícios resistidos para grandes grupos musculares (American College of Sports Medicine ACSM, 2007). As sessões de treino foram realizadas com uma frequência semanal de três vezes, com uma duração de 60 minutos e por um período de 12 semanas. No programa, deste grupo, foi ainda incluído exercícios de alongamentos associados a exercícios respiratórios. Ao término da aplicação dos protocolos, a amostra foi reavaliada.

\section{Análise estatística}

Após confirmação dos pressupostos de normalidade das variáveis em estudo pelo teste Kolmogorov-Smirnov, foi efectuada a comparação das médias pela análise de variância (ANOVA) com Post-Hoc de Tukey. Para verificar os efeitos dos procedimentos terapêuticos dentro do mesmo grupo foi aplicado o teste $t$ para amostras emparelhadas. Foram ainda investigadas associações entre variáveis com o coeficiente de correlação de Pearson. O nível mínimo de significância adaptado foi de $5 \%$. Os resultados são apresentados como médias e desvios-padrão (DP). 


\section{RESULTADOS}

A comparação dos valores médios para as variáveis antropométricas massa corporal, estatura e IMC não foram diferentes significativamente entre os grupos $(p>.05)$ no período pré-terapêutica, assim como os valores médios para PImáx e PEmáx (tabela 1). No entanto, observou-se que a idade média de G3 (48.75 \pm 13.71 anos) foi estatisticamente diferente, $F_{(2,5.37)}=625.87, p=.008$, quando comparado ao G1 (57.37 \pm 8.10 anos) e ao G2 (60.31 \pm 8.94 anos). A média da estatura para os grupos G1, G2 e G3 foram $1.52 \pm .04,1.53$ \pm .06 e $1.55 \pm .05$, respectivamente.

Tabela 1

Valores médios (M) e desvio padrão (DP) para mulheres obesas pré e pós aplicação de diferentes terapêuticas

\begin{tabular}{lcccccc}
\hline & \multicolumn{2}{c}{ Sedentárias } & \multicolumn{2}{c}{ Activas } & \multicolumn{2}{c}{ Reabilitação Pulmonar } \\
& Pré & Pós & Pré & Pós & Pré & Pós \\
& $M \pm D P$ & $M \pm D P$ & $M \pm D P$ & $M \pm D P$ & $M \pm D P$ & $M \pm D P$ \\
\hline $\mathrm{MC}$ & $89.4 \pm 14.3$ & $89.4 \pm 14.3$ & $89.2 \pm 16.3$ & $89.2 \pm 16.3$ & $83.3 \pm 12.1$ & $81.3 \pm 1.8^{* *}$ \\
$\mathrm{IMC}$ & $38.3 \pm 5.9$ & $38.3 \pm 5.9^{*}$ & $38.0 \pm 7.7$ & $38.0 \pm 7.7$ & $34.7 \pm 3.8$ & $33.8 \pm 3.9$ \\
$\mathrm{PI}_{\text {máx }}$ & $-67.2 \pm 23.2$ & $-87.8 \pm 25.9^{* *}$ & $-81.5 \pm 24.7$ & $-95.4 \pm 19.0^{* *}$ & $-69.8 \pm 21.4$ & $-78.3 \pm 21.4^{*}$ \\
$\mathrm{PE}_{\text {máx }}$ & $72.5 \pm 20.9$ & $91.4 \pm 20.9^{* *}$ & $73.5 \pm 25.4$ & $84.6 \pm 17.6^{* *}$ & $74.5 \pm 28.2$ & $94.0 \pm 24.7^{* *}$ \\
${ }^{*} p<.05$ para comparação intra-grupo, ${ }^{* *} p<.01$ para comparação intra-grupo; $\mathrm{MC}=$ massa corporal $(\mathrm{kg}), \mathrm{IMC}=$ índice \\
de massa corporal $\left(\mathrm{kg} / \mathrm{m}^{2}\right)$, PImáx $=$ pressão inspiratória máxima, PEmáx = pressão expiratória máxima
\end{tabular}

A análise dos resultados no período pósterapêutico demonstrou que as actividades desenvolvidas não desencadearam estímulos suficientes para promover alterações significativas na massa corporal das voluntárias, porém, o IMC do G1 foi significativamente diferente em relação aos G2 e G3, $F_{(2,3.51)}=117.03, p=.03$ (ver tabela 1 ). Quanto aos valores observados para a PImáx e PEmáx pós-terapêutica não foi constatado que a prática de actividades físicas, realizadas sem uma planificação das intensidades, tenha influência nos ganhos do G2. O que se constatou, após decorrido os programas terapêuticos, foi que a homogeneidade entre os grupos permaneceu. Onde observa-se os respectivos valores, $F_{(2,2.81)}=925.16, p=.07 \mathrm{e}$, $\mathrm{F}_{(2, .76)}=460.54, p=.47$.

Ao serem comparadas as médias obtidas intra-grupo após 12 semanas de programa de reabilitação pulmonar e de 7 dias de aplicação do incentivador respiratório, observa-se um ganho tanto para a PIMax, como para a PEmáx nos grupos G1 e G2. Comportamento similar foi observado para o grupo G3, demonstrado a eficácia das terapêuticas na melhoria das respectivas pressões estáticas (tabela 1).
Ao se correlacionar as variáveis antropométricas e as pressões respiratórias estáticas máximas observou-se que as mesmas não apresentaram correlações significativas no período pré-tratamento. No entanto, no período pós-tratamento, foram confirmados valores de correlação fracos entre a PImax e IMC $(r=-.33, p=.01)$ para todos os grupos.

\section{DISCUSSÃO}

A obesidade é uma enfermidade de dimensões socioculturais que deve ser analisada dentro da sua complexidade. Permitindo, assim, a aplicação de métodos que procurem explicar o comportamento ou influência de cada variável a ela relacionada. Talvez, ao se adaptar uma estratégia que permita estabelecer ou adequar protocolos de intervenção individualizados, possa-se hoje obter melhores respostas (Goldberger et al., 2002; Pakenas, Souza Junior, \& Pereira, 2007). No entanto, a aplicação da teoria da complexidade, ao se tentar estabelecer a geometria fractal, pode ser vista como um problema maior em função da flutuação sociocultural da enfermidade dentro do seu eixo etiológico (Pakenas et al., 2007) já que suas multifaces implicariam em 
incontáveis variáveis intervenientes (Cataneo, Carvalho, \& Galindo, 2005; Ferreira \& Magalhães; 2005; Pitanga \& Lessa, 2005).

O presente estudo teve por objectivo verificar os efeitos de diferentes terapêuticas sobre as pressões respiratórias estáticas máximas em mulheres obesas. Foram aplicados procedimentos que resultaram em alterações positivas na mecânica respiratória e que permitissem o restabelecimento dos parâmetros funcionais como respostas aos protocolos aplicados. Sem que contudo, fossem alterados aspectos morfológicos na amostra. Deve-se salientar que os resultados do IMC obtidos, no presente estudo, encontram-se acima, quando comparados aos descritos para mulheres com média de idade de $68.91 \pm 5.43$ anos, residentes na cidade de São Paulo (Barbosa, Santarém, Jacob Filho, \& Marucci, 2001), o que corrobora com os estudos de Menezes e Marucci (2007) e Rezende, Rosado, Priore e Franceschini (2006) que apontam a influência direta da cultura no âmbito da alimentação em imigrantes nordestinos nas favelas da cidade do Rio de Janeiro. Dentro desta perspectiva, as voluntárias do presente estudo residem na cidade de Natal - RN, o que permite inferir que tais hábitos alimentares estejam ligados aos valores obtidos para IMC.

O ponto de relevância a ser destacado, no presente estudo, é a redução do IMC no grupo $\mathrm{G} 3$, resultados que divergem dos apresentados no estudo de Barbosa et al. (2001), onde mulheres idosas saudáveis foram submetidas ao programa de treino resistido por 10 semanas, com uma frequência de três dias, e não apresentaram alterações significativas nas dimensões corporais após o período de treino. $O$ que poderia explicar os resultados observados nesse estudo seria a combinação de actividades aeróbias e anaeróbias aliadas aos exercícios respiratórios, assim como um período de treino superior ao que foi aplicado no estudo citado. Essas alterações ocorridas na massa corporal do grupo G3, estão directamente relacionadas à aplicação de estímulos adequados, os quais ajudaram a promover modificações morfológicas, embora o programa tenha tido uma característica linear. Tal característica linear pode ser observada também nas actividades realizados pelo grupo G1, porém, infere-se que as adaptações crónicas ocorridas passassem a exigir a implicação de novos estímulos (intensidade ou volume) para que ocorressem novas alterações (Kesaniemi et al., 2001). Esses resultados ratificam a importância da implementação de equipas multidisciplinares, nos centros de saúde, na orientação e prescrição de actividades físicas para a população. $\mathrm{O}$ que permite promover alterações na percepção da qualidade de vida de indivíduos acometidos por enfermidades degenerativas. Tais inferências podem ser apoiadas nos resultados obtidos no estudo de Mota, Ribeiro, Carvalho e Matos (2006), onde foi constatado que as percepções da qualidade de vida de idosos engajados em programas de actividade física eram significativamente diferentes de seus pares que não estavam engajados. $\mathrm{O}$ que não pode ser esquecido é a importância da actividade física nos aspectos relacionados aos quadros depressivos, principalmente em mulheres com característica de obesidade, como as do presente estudo (Gumarães \& Caldas, 2006).

O ganho significativo nas pressões inspiratórias e expiratórias estáticas máximas em todos os grupos, sem que fosse demonstrada uma relação directa entre as medidas e os parâmetros antropométricos, se contrapõem aos apontamentos de Maeda (2004). Mas ao mesmo tempo, Robergs e Roberts (2002) citam que não há uma relação entre a mecânica ventilatória e a melhoria da aptidão cardiorrespiratória. $O$ que foi inversamente observado no grupo G3, corroborando com os apontamentos de Powers e Howley (2000) que descrevem que a prática de exercícios físicos de intensidade leve a moderado pode exercer influência sobre as capacidades e perfusão pulmonares. Os resultados observados para as pressões estáticas máximas, no presente estudo, 
demonstram que a aplicação de exercícios para a musculatura respiratória proporciona melhorias consistentes em indivíduos que apresentem quadro de obesidade.

A prática de exercícios aeróbios, alongamentos e exercícios ventilatórios diafragmáticos forneceram resultados que reequilibraram a musculatura inspiratória e expiratória minimizando as alterações decorrentes da debilidade muscular. Estimulando a actividade diafragmática e assim promovendo aumento do volume corrente (Robergs \& Roberts, 2002). Em estudo que relacionou a prática de exercícios globais associados aos exercícios respiratórios, realizados em um programa de reabilitação através da Reeducação Postural Global - RPG, em um período de 8 semanas, em jovens saudáveis e sedentários ocasionou melhora significativa nas pressões estáticas máximas e na mobilidade tóraco-abdominal (Moreno, Catai, Teodori, Borges, Castro Cesar, \& Silva, 2007), o que corrobora com os resultados observados no presente estudo, para o grupo G3.

A ausência de correlação observada, no presente estudo, entre o IMC e a PImáx ou a PEmáx para os períodos pré e pós-terapêutico, divergem dos estudos em que o aumento da adiposidade com a idade, principalmente na região visceral, estaria correlacionado com a redução das pressões respiratórias estáticas (Maeda, 2004; Menezes \& Marucci, 2007). No entanto, os dados obtidos nesse estudo corrobora com Azeredo (2000), o qual descreve a relação entre o acúmulo de gordura nas alterações da mecânica respiratória. O que pode explicar os resultados observados no presente estudo quanto à redução da mecânica respiratória observada no período préterapêutico. Sendo este relacionado não apenas com a restrição do movimento de expansibilidade torácica, em detrimento do acúmulo de gordura, mas também pelo facto da redução da força muscular, ocorrida no processo degenerativo, estar relacionada ao sedentarismo e/ou a baixa intensidade das actividades físicas. Por outro lado, embora o IMC possa reflectir a quantidade de massa gorda do indivíduo, não a representa directamente. Logo, também esse viés poderia explicar a ausência de correlação observada.

\section{CONCLUSÃO}

Em conclusão, os dados obtidos no presente estudo demonstram que, tanto a terapêutica do incentivador respiratório como um programa de reabilitação pulmonar favorecem a melhoria significativa das pressões respiratórias estáticas máximas de mulheres obesas, com diferentes níveis de aptidão física. Quanto à correlação entre as pressões respiratórias estáticas máximas e as variáveis antropométricas não foram observados resultados significativos. Assim, permitimo-nos destacar, a importância da implementação de programas de exercícios físicos supervisionados, por uma equipe multidisciplinar, onde seja possível a combinação de procedimentos que tanto permita a melhoria das capacidades funcionais, como também a aquisição de um estilo de vida mais saudável.

\section{REFERÊNCIAS}

American College of Sports Medicine - ACSM (2007). Diretrizes do ACSM para os testes de esforço e sua prescrição ( $7^{\mathrm{a}}$ ed.). Rio de Janeiro: Guanabara Koogan.

Azeredo, C. A. C. (2000). Fisioterapia respiratória no hospital geral: Expansão, reexpansão e recrutamento Alveolar. São Paulo: Manole.

Barbosa, A. R., Santarém, J. M., Jacob Filho, W., \& Marucci, M. F. N. (2001). Composição corporal e consumo alimentar de idosas submetidas a treinamento contra resistência. Revista de Nutrição, 14,177-183.

Cataneo, C., Carvalho, A. M. P., \& Galindo, E. M. C. (2005). Obesidade e aspectos psicológicos: Maturidade emocional, auto-conceito, locus de controle e ansiedade. Psicologia: Reflexão e Crítica, 18, 39-46.

Domingos, B. N. C. (2003). Influência do peso corporal sobre as pressões respiratórias máximas nas posições sentada, deitada e em pé. Revista Brasileira de Fisioterapia, 3, 217-222. 
Ferreira, V. A., \& Magalhães, R. (2005). Obesidade e pobreza - O aparente paradoxo: Um estudo com mulheres da Favela da Rocinha, Rio de Janeiro, Brasil. Cadernos de Saúde Pública, 21, 1792-1800.

Godoy, R. F., Teixeira Paulo, J. Z., Becker Júnior, B., Michelli M., \& Godoy, D. V. (2009). Long-term repercussions of a pulmonary rehabilitation program on the indices of anxiety, depression, quality of life and physical performance in patients with COPD. Jornal Brasileiro de Pneumologia, 35, 129-136.

Goldberger, A. L., Amaral, L. A. N., Hausdorff, J. M., Ivanov, P. C., Peng, C. K., \& Stanley, H. E. (2002). Fractal dynamics in physiology: Alterations with disease and aging. Proceedings of the National Academy of Sciences, 99, 24662472.

Guimarães, H. P., Avezum, A., \& Piegas, L. S. (2006). Obesidade abdominal e síndrome metabólica. Revista da Sociedade de Cardiologia do Estado de São Paulo, 16, 41-45.

Guimarães, J. M. N., \& Caldas, C. P. (2006). A influência da atividade física nos quadros depressivos de pessoas idosas: Uma revisão sistemática. Revista Brasileira de Epidemiologia, 9, 481-492.

Kesaniemi, Y. K., Daforth, E., Jensen, M. D., Kopelman, P. G., Lefebvre, P., \& Reeder, B. A. (2001). Dose-response issues concerning physical activity and health: An evidence-based symposium. Medicine $\mathcal{E}$ Science in Sports $\mathcal{E}$ Exercise, 33, S351-S358.

Maeda, S. S. (2004) Prevalência global e tendências. In O.M.S. (Ed.), Obesidade: Prevenindo $e$ controlando a epidemia global. São Paulo: Roca.

Menezes, T. N., \& Marucci, M. F. N. (2007). Perfil dos indicadores de gordura e massa muscular corporal dos idosos de Fortaleza, Ceará, Brasil. Cadernos de Saúde Pública, 23, 2887-2895.

Moreno, M. A., Catai, A. M., Teodori, R. M., Borges, B. L. A., Castro Cesar, R. M., \& Silva, E. (2007). Efeitos de um programa de alongamento muscular pelo método de reeducação postural global sobre a força muscular respiratória e a mobilidade toracoabdominal de homens jovens sedentários. Jornal Brasileiro de Pneumologia, 33(6), 679-686.

Mota, J., Ribeiro, J. L., Carvalho, J., \& Matos, M. G. (2006). Atividade física e qualidade de vida associada à saúde em idosos participantes e não participantes em programas regulares de atividade física. Revista Brasileira de Educação Física e Esporte, 20, 219-25.

Paisani, D. N., Chiavegato, L. D., \& Faresin, S. M. (2005). Volumes, capacidades pulmonares e força muscular respiratória no pós-operatório de gastroplastia. Jornal Brasileiro de Pneumologia, $31,124-132$.

Pakenas, A., Souza Junior, T. P., \& Pereira, B. (2007). Dinâmica não-linear e exercício físico: conceitos e aplicações. Revista Brasileira de Medicina do Esporte, 13, 331-335.

Parreira, V. F., França, D. C., Zampa, C. C., Fonseca, M. M., Tomich, G. M., \& Britto, R. R. (2007). Pressões respiratórias máximas: valores encontrados e preditos em indivíduos saudáveis. Revista Brasileira de Fisioterapia, 5, 361-368.

Pitanga, F. J. G., \& Lessa, I. (2005). Prevalência e fatores associados ao sedentarismo no lazer em adultos. Cadernos de Saúde Pública, 21, 870-877.

Powers, S. K., \& Howley, E. T. (2000). Fisiologia do exercício: Teoria e aplicação ao condicionamento e ao desempenho ( $2^{\mathrm{a}}$ ed.). São Paulo: Manole

Rezende, F. A. C., Rosado, L. E. F., Priore, S. E., \& Franceschini, S. C. C. (2006). Aplicabilidade de equações na avaliação da composição corporal da população brasileira. Revista de Nutrição, 19, 357-367.

Robergs, R. A., \& Roberts, S. O. (2002). Princípios fundamentais de fisiologia do exercício: Para aptidão, desempenho e saúde. São Paulo: Phorte.

Rocca S. V. S., Tirapegui J., Melo, C. M., \& Ribeiro, S. M. L. (2008). Efeito do exercício físico nos fatores de risco de doenças crônicas em mulheres obesas. Revista Brasileira de Ciências Farmacêuticas, 44, 185-192.

Romanini, W., Muller, A. P., Carvalho, K. A. T., Olandoski, M., Faria-Neto, J. R., Mendes, F. L. et al. (2007). Os efeitos da pressão positiva intermitente e do incentivador respiratório no pós-operatório de revascularização miocárdica. Arquivo Brasileiro de Cardiologia, 89, 105-110.

Romero, C. E. M., \& Zanesco, A. (2006). O papel dos hormônios leptina e grelina na gênese da obesidade. Revista de Nutrição, 19, 85-91.

Sociedade Brasileira de Pneumologia e Tisologia SBPT (2002). Diretrizes para testes de função pulmonar. Jornal Brasileiro de Pneumologia, 28(S3), S1-S238. 